\title{
Response of Disordered Polymer Networks to External Fields: Regular Lattices Built from Complex Subunits
}

\author{
Andrew A. Gurtovenko*,t,t and Alexander Blumen ${ }^{\dagger}$ \\ Theoretische Polymerphysik, Universität Freiburg, Hermann-Herder-Str. 3, \\ D-79104 Freiburg, Germany, and Institute of Macromolecular Compounds, Russian Academy of \\ Sciences, Bolshoi Prospect 31, V.O., St. Petersburg, 199004 Russia \\ Received October 24, 2001; Revised Manuscript Received J anuary 30, 2002
}

\begin{abstract}
Based on recent experiments, inhomogeneous polymer networks can be viewed as densely cross-linked regions embedded in more dilute surroundings. Here we put forward systems that model such situations, namely regular lattices built from complex subunits. The model takes into account both the disorder inside the subunits (cross-link aggl omerations) and the connectivity between them. Combining analytical and numerical methods, we propose a general approach for determining the Rouse dynamics of such systems. Exemplarily, we apply this approach to two-dimensional lattices built from small-world Rouse networks and evaluate the model's mechanical and dielectric response to external fields. We find that the networks show interesting relaxation features and an unusual "plateau-type" behavior in the intermediate frequency (time) domain, which lies between the modes of the disordered subunits and those of the regular lattice. This behavior is di rectly related to the disorder inside the cross-link agglomerations and may be readily detected through appropriate mechanical and dielectric experiments.
\end{abstract}

\section{Introduction}

One of the basic challenges in polymer theory is to deduce how the topology of polymers affects their dynamical properties. In this work we focus on polymer networks as a very important class of complex systems; in such networks the macromolecules are connected with each other by means of chemical cross-links. Because cross-linking often occurs randomly, usually polymer networks show quite a disordered structure, a fact which renders their theoretical treatment difficult. Up to now most theor etical approaches have focused on networks that are either regularly built or self-similar, the idea being that both cases allow to use the symmetries inherent in the underlying geometry. Examples for the first case are simple topologies, such as regular lattices, star polymers, or dendrimers; examples for the second kind are percolating clusters.

Many real cross-linked polymers do not belong to any of these classes. The reason for this is quite subtle, and it may be inherent in the preparation of cross-linked samples, where different kinetic factors influence the formation of the networks; such networks may (due to their durable chemical bonds) retain for a very long time the memory of their preparation. That one is indeed confronted with quite complex systems is well-documented experimentally. Thus, small-angle neutron scattering experiments ${ }^{1,2}$ have shown that in statistical gels the local density of cross-links varies widely: The random arrangement of cross-links in the gel leads to local regions (clusters), whose cross-link density is much higher than on the average. Therefore, under contact with an excess of solvent, the solvent is mainly absorbed by the regions between the clusters, and the gel swells heterogeneously. ${ }^{1,2}$ Moreover, dynamic and static light

† Universität Freiburg.

₹ Russian Academy of Sciences.

* To whom correspondence should be addressed. Current address: Theoretische Polymerphysik, Universität Freiburg, Hermann-Herder-Str. 3, D-79104 Freiburg, Germany. scattering experiments on hydrogels have also revealed highly heterogeneous structures. ${ }^{3-5}$ As demonstrated in the recent work of Oppermann and co-workers on polyacrylamide hydrogels, ${ }^{5}$ the gel structure can be visualized as being built from densely cross-linked clusters (cross-link agglomerations) distributed in surroundings whose cross-link density is lower. That, indeed, the clusters are themsel ves interconnected (for sufficiently dense polymer systems) is reflected in the nonzero value of the equilibrium modulus of polymer networks and gels. ${ }^{6}$ In fact, the networks appear to be inhomogeneous (stochastic) on small length scales, but they are rather homogeneous on large length scales.

The problem that we face is to devel op models for such network systems, models which reflect their disordered structure but which still allow to determine their dynamical properties in a not-too-intricate fashion. For this we prefer to work in the framework of generalized Gaussian structures ${ }^{7,8}$ (GGS), which are extensions of the classical Rouse model ${ }^{9,10} \mathrm{~A}$ simple way to take into account the structural features mentioned above is to "decorate" a regular lattice (say square or cubic) by replacing its bonds with subunits (cross-link agglomerations) having complex internal structures. Such an approach models some of the disorder inherent in realistic networks; on the other hand, decorating regular structures with complex subunits allows us to use methods developed in solid-state physics in the treatment of lattices made up of subunits. We hasten to notice that polymer networks do not display in general any translational symmetry. However, translational symmetry is not required: The idea is that for generalized Gaussian structures (GGS) the corresponding Langevin equations have a topological symmetry mathematically very akin to the translational symmetry of perfectly regular lattices. This fact all ows to considerably simplify the problem, since we are then able to reduce the determination of the normal modes of our structures to the diagonalization of relatively small matrices, where the large-scale symmetry is taken into 

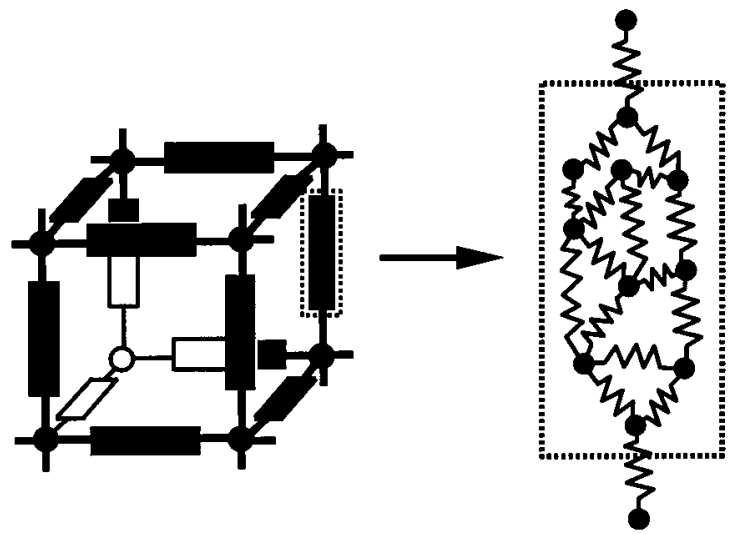

Figure 1. Three-dimensional cubic lattice built from complex subunits with arbitrary internal structure. Shown is one elementary cell of the regular lattice and the internal structure of one subunit (surrounded by a dashed line).

account through judiciously chosen boundary conditions, reflected in the appearance of corresponding phase factors.

The paper is organized as follows. In the next section we introduce our model for the polymer networks and gels mentioned, namely regular lattices built from complex subunits. In section 2 we also discuss the dynamical features which we are interested in, given that the usual dynamic experiments measure the mechanical and dielectric response of polymer networks to external fields. Section 3 is devoted to solving the dynamical problems which arise in this context for networks built from complex subunits. Exemplarily, in section 4 we apply our general formal ism to di sordered polymer networks using as subunits small-world Rouse networks (SWRN), which we introduce and discuss. In fact, SWRNs are quite disordered, a fact which leaves its mark on the experimental patterns obtained through mechanical and dielectric relaxation measurements. Finally, in section 5 we end with a short summary and conclusions.

\section{Mechanical and Dielectric Relaxation of Generalized Gaussian Structures}

To model the gels and networks discussed in the Introduction, we start from topologically regular lattices; then we replace the bonds connecting their vertexes with complex subunits, which may have arbitrary internal architectures. In this way we take into account the two basic features of the polymeric gels discussed above: We can account for the disorder inside the crosslink agglomerations through the judicious choice of subunits; the global connectivity of the network (which on long length scales is quite regular) is given by the regular lattice. In Figure 1 we display one possible elementary cell of such a network, where we have, for simplicity, depicted it as a part of a three-dimensional network, related to a simple cubic lattice. As will be evident in the following, one may use other basic lattices. Furthermore, we take all the subunits to have the same topological internal structure. In this case, as we proceed to show, the long-range regularity of the network allows us to simplify the problem using Floquet's theorem (whose special case in solid-state physics is Bloch's theorem ${ }^{11-13}$ ).

We perform our study in the framework of the Rouse model ${ }^{9,10}$ and of its extensions called generalized Gaussian structures 7,8 (GGS). A GGS (full network) consists of beads connected to each other by springs (with elasticity constant $K$ ) and subject to the friction constant $\xi$, which arises from the interaction with the solvent or with the effective viscous medium. ${ }^{10}$ In the Langevin framework, the position vector $\mathbf{R}_{l}(\mathrm{t})$ of the Ith bead of the network (GGS), subject to the external force $\mathbf{F}_{\mid}(\mathrm{t})$, obeys

$$
\xi \frac{\mathrm{d} \mathbf{R}_{l}(\mathrm{t})}{\mathrm{dt}}+\mathrm{K} \sum_{\mathrm{m}=1}^{\mathrm{N}_{\text {tot }}} \mathrm{A}_{I \mathrm{~m}} \mathbf{R}_{\mathrm{m}}(\mathrm{t})=\mathbf{F}_{\mid}(\mathrm{t})+\zeta \mathbf{w}_{\mid}(\mathrm{t})
$$

where $\mathbf{A}=\left\{A_{\mid m}\right\}$ is the connectivity matrix of the GGS (see refs 7 and 14 for details), $\zeta \mathbf{w}_{l}(\mathrm{t})$ is the thermal noise (here assumed to be Gaussian, with zero mean value), and $\mathrm{N}_{\text {tot }}$ is the total number of elements (beads) in the system considered. The linear system of differencedifferential equations, eq 1, can be completely solved through the diagonalization of the matrix $\mathbf{A}$, a procedure which involves in general determining both the eigenvalues and the eigenfunctions of $\mathbf{A}$. As shown in the next section, the procedure simplifies for structures akin to those of Figure 1.

Let us outline now the measurable relaxation quantities, which we are interested in and which are experimentally accessible. In general, mechanical and dielectric relaxations are well-established techniques in polymer physics. In typical mechanical experiments one measures the complex (shear) modulus $\mathrm{G}^{*}(\omega)$ as response to a harmonic strain field. For the structures considered here (GGS) one has for $\mathrm{G}^{\prime}(\omega)$, the real, and for $\mathrm{G}^{\prime \prime}(\omega)$, the imaginary component of the complex dynamic modulus $\mathrm{G}^{*}(\omega):^{10}$

$$
\mathrm{G}^{\prime}(\omega)=\nu \mathrm{k}_{\mathrm{B}} \mathrm{T} \frac{1}{\mathrm{~N}_{\text {tot }}=21+\left(\omega \tau_{0} / 2 \lambda_{\mathrm{i}}\right)^{2}}
$$

and

$$
\mathrm{G}^{\prime \prime}(\omega)=\nu \mathrm{k}_{\mathrm{B}} \mathrm{T} \frac{1}{\mathrm{~N}_{\text {tot }}} \sum_{\mathrm{i}=2 \mathrm{~N}}^{\mathrm{N}_{\text {tot }}} \frac{\omega \tau_{0} / 2 \lambda_{\mathrm{i}}}{1+\left(\omega \tau_{0} / 2 \lambda_{\mathrm{i}}\right)^{2}}
$$

In eqs 2 and $3 v$ is the number of polymer segments (beads) per unit volume, $\tau_{0}=\xi / K$, and the $\lambda_{\text {i }}$ are the eigenvalues of the connectivity matrix $\mathbf{A}$ of the GGS. In eqs 2 and 3 we have neglected the vanishing eigenvalue denoted by $\lambda_{1}=0$, which corresponds to the translation of the whole GGS and which may influence $\mathrm{G}^{\prime \prime}(\omega)$ only in the very close vicinity of $\omega=0$. The components $\mathrm{G}^{\prime}(\omega)$ and $\mathrm{G}^{\prime \prime}(\omega)$ are usually called storage and loss modulus, respectively. ${ }^{6}$

Now the dynamic modulus $\mathrm{G}^{*}(\omega)$ is related in the time domain to the relaxation modulus $\mathrm{G}(\mathrm{t})$ (the response of the stress to a shear jump) through

$$
\mathrm{G}^{*}(\omega)=\mathrm{i} \omega \int \mathrm{G}(\tau) \exp (-\mathrm{i} \omega \tau) \mathrm{d} \tau
$$

From eqs 2 and $3 \mathrm{G}(\mathrm{t})$ obeys

$$
\mathrm{G}(\mathrm{t})=v \mathrm{k}_{\mathrm{B}} \mathrm{T} \frac{1}{\mathrm{~N}_{\text {tot }}} \sum_{\mathrm{i}=2}^{\mathrm{N}_{\text {tot }}} \exp \left(-\mathrm{t} \frac{2 \lambda_{\mathrm{i}}}{\tau_{0}}\right)
$$

Furthermore, the mechanical relaxation of viscoelastic materials can be alternatively described (apart from moduli) by means of the relaxation spectrum $\mathrm{H}(\tau)$ (i.e., 
the distribution function of relaxation times $\tau$ on a logarithmic scale), which is connected with the relaxation modulus $\mathrm{G}(\mathrm{t})$ through $^{6}$

$$
\mathrm{G}(\mathrm{t})=\int_{0}^{\infty} \mathrm{H}(\tau) \exp (-\mathrm{t} / \tau) \mathrm{d} \ln \tau
$$

Note that the factor 2 in the exponents of eq 5 (equivalently, in the relaxation times $\tau_{\mathrm{i}}=\tau_{0} / 2 \lambda_{\mathrm{i}}$ ) arises from the second moment of the displacements involved in computing the stress; see the evaluation of $\mathrm{G}^{*}(\omega)$ and, correspondingly, of $\mathrm{G}(\mathrm{t})$ in ref 10 .

The dielectric relaxation forms are based on the frequency-dependent complex dielectric susceptibility, $\epsilon^{*}(\omega)$. Now one focuses usually on

$$
\Delta \epsilon^{*}(\omega)=\frac{\epsilon^{*}(\omega)-\epsilon_{\infty}}{\epsilon_{0}-\epsilon_{\infty}}
$$

where $\epsilon_{0}$ and $\epsilon_{\infty}$ denote the limiting low- and highfrequency diel ectric constants, respectively. In general, subjecting polar molecules embedded in nonpolar solvents to an alternating electric field $\mathrm{E}=\mathrm{E}_{0} \exp (\mathrm{i} \omega \mathrm{t})$ leads to the $\Delta \epsilon^{*}(\omega)$ response: ${ }^{15}$

$$
\Delta \epsilon^{*}(\omega) \simeq \int_{0}^{\infty}\left(-\frac{\mathrm{d}}{\mathrm{dt}} \mathrm{C}_{0}(\mathbf{M} ; \mathrm{t})\right) \exp (-\mathrm{i} \omega \mathrm{t}) \mathrm{dt}
$$

when the local fields are not important. In eq 8 the quantity $\mathrm{C}_{0}(\mathbf{M}$; $\mathbf{t})$ is the normalized autocorrelation function of the total dipole moment $\mathbf{M}(\mathrm{t})$ of the system,

$$
\mathrm{C}_{0}(\mathbf{M} ; \mathrm{t})=\frac{\langle\mathbf{M}(0) \mathbf{M}(\mathrm{t})\rangle}{\left\langle\mathbf{M}^{2}(0)\right\rangle}
$$

We focus here on the dielectric response of GGSs, which possess dipole moments directed along their bonds (type A according to Stockmayer's classification). ${ }^{16-18}$ We assign a longitudinal dipole moment $\mathbf{~ m}_{\mathrm{j}}$ to each bond $\mathrm{j}$ of the GGS; the magnitude of each $\mathbf{m}_{\mathrm{j}}$ is directly proportional to the length of the jth bond, but its orientation is arbitrary. Therefore, the total dipole moment $\mathbf{M}(\mathrm{t})$ of the GGS is given by

$$
\mathbf{M}(\mathrm{t})=\sum_{\mathrm{j}=1}^{\mathrm{N}_{\mathrm{b}}} \mu_{\mathrm{j}} \mathbf{P}_{\mathrm{j}}(\mathrm{t})
$$

where $N_{b}$ is the number of bonds of the GGS, $\mu_{\mathrm{j}}=\mathrm{I}_{\mathrm{j}} \mu$ is the dipole moment per unit length, $\mathrm{l}_{\mathrm{j}} \in\{+1,-1\}$ gives the orientation, and $\mathbf{P}_{\mathrm{j}}=\mathbf{R}_{\mathrm{l}}-\mathbf{R}_{\mathrm{m}}$ is the vector of the jth bond between the Ith and the mth GGS beads. We consider here the special situation in which the $l_{j}$, i.e., the orientations of the dipole moments $\left\{\mathbf{m}_{\mathbf{j}}\right\}$ along the bonds, are random and uncorrelated. Then one has, averaged over all possible orientation distributions,

$$
\begin{array}{r}
\langle\langle\mathbf{M}(0) \mathbf{M}(\mathrm{t})\rangle\rangle_{\text {or }}=\sum_{\mathrm{j}=1}^{\mathrm{N}_{\mathrm{b}}} \sum_{\mathrm{k}=1}^{\mathrm{N}_{\mathrm{b}}} \mu^{2}\left\langle\mathrm{I}_{\mathrm{j}} \mathrm{I}_{\mathrm{k}}\right\rangle_{\text {or }}\left\langle\mathbf{P}_{\mathrm{j}}(0) \mathbf{P}_{\mathrm{k}}(\mathrm{t})\right\rangle= \\
\mu^{2} \sum_{\mathrm{j}=1}^{\mathrm{N}_{\mathrm{b}}}\left\langle\mathbf{P}_{\mathrm{j}}(0) \mathbf{P}_{\mathrm{j}}(\mathrm{t})\right\rangle
\end{array}
$$

since $\left\langle\mathrm{l}_{\mathrm{j}} \mathrm{I}_{\mathrm{k}}\right\rangle_{\mathrm{or}}=\delta_{\mathrm{jk}}$. The combination of coordinates of GGS beads involved in the quantity $\langle\langle\mathbf{M}(0) \mathbf{M}(\mathrm{t})\rangle\rangle_{\text {or, }}$ eq 11 , is directly related to that in the potential energy $U(\{\mathbf{R}\})$ of the GGS, which enters in the Langevin equation:

$$
\mathrm{U}(\{\mathbf{R}\})=\frac{\mathrm{K}^{\mathrm{N}}}{2} \sum_{\mathrm{j}=1}^{\mathrm{N}_{\mathrm{b}}} \mathbf{P}_{\mathrm{j}}^{2}
$$

Using this fact, one can reduce the normalized autocorrelation function of the total dipole moment $\mathrm{C}_{0}(\mathbf{M} ; \mathbf{t})$ to the following simple form (see the detailed derivation in the Appendix):

$$
\mathrm{C}_{0}(\mathbf{M} ; \mathrm{t})=\frac{1}{\mathrm{~N}_{\text {tot }}} \sum_{\mathrm{i}=1}^{\mathrm{N}_{\text {tot }}} \exp \left(-\lambda_{\mathrm{i}} \mathrm{t} / \tau_{0}\right)
$$

where $\mathrm{N}_{\text {tot }}$ and the $\lambda_{\mathrm{i}}$ are as before. Note that the approach presented here is very similar to that developed for the dielectric relaxation of regular meshlike networks built from macromolecules with Iongitudinal dipole moments ${ }^{19}$ as well as to that for the dielectric relaxation of small-world Rouse networks. ${ }^{20}$

Inserting now eq 13 in eq 8 leads to the dielectric susceptibility $\Delta \epsilon^{*}$; expressing it in terms of its real and imaginary parts, $\Delta \epsilon^{*}=\Delta \epsilon^{\prime}-\mathrm{i} \Delta \epsilon^{\prime \prime}$, one finds

$$
\Delta \epsilon^{\prime}(\omega)=\frac{1}{N_{\text {tot }}} \sum_{=21}^{N_{\text {tot }}} \frac{1}{1+\left(\omega \tau_{0} / \lambda_{\mathrm{i}}\right)^{2}}
$$

and

$$
\Delta \epsilon^{\prime \prime}(\omega)=\frac{1}{N_{\text {tot }}=2} \sum_{\text {tot }}^{N_{1}} \frac{\omega \tau_{0} / \lambda_{\mathrm{i}}}{\left.1 \omega \tau_{0} / \lambda_{\mathrm{i}}\right)^{2}}
$$

For the model considered here we thus have a straightforward correspondence between the mechanical and dielectric relaxation forms; from eqs 2, 3, 14, and 15 one sees readily that

$$
\Delta \epsilon^{\prime}(\omega)=1-\mathrm{G}^{\prime}(2 \omega) / v \mathrm{k}_{\mathrm{B}} \mathrm{\top}
$$

and

$$
\Delta \epsilon^{\prime \prime}(\omega)=\mathrm{G}^{\prime \prime}(2 \omega) / v \mathrm{k}_{\mathrm{B}} \mathrm{T}
$$

hold. One may furthermore note that the dielectric susceptibility, eqs $13-15$, is determined by relaxation times which are twice larger than those appearing in the expressions of mechanical relaxation, eqs 2,3 , and 5. We like to emphasize that for calculating $G(t)$, $\mathrm{G}^{*}(\omega)$, and $\Delta \epsilon^{*}(\omega)$ we need only the eigenvalues $\lambda_{\mathrm{i}}$ (or the relaxation times $\tau_{\mathrm{i}}$ ) of the GGS, but not the corresponding eigenfunctions. In fact, such relaxation characteristics (which are determined by the eigenvalues only) simplify the solution of the dynamical problems considered here.

\section{Lattices Consisting of Subunits}

In this section we consider the dynamics of networks consisting of a topologically regular lattice, decorated with subunits (see Figure 1). The simplest realization for the subunits between lattice vertexes is the standard linear Rouse chain. In this case the problem can be solved analytically for regular 2D and 3D lattices. ${ }^{21-24}$ Basic feature is that for such Rouse chain subunits the relaxation spectrum $\mathrm{H}(\tau)$ of the networks, eq 6 , displays two characteristic domains; these correspond to the small-scale (intrachain) motions and to the large-scale (interchain) motions of the network. ${ }^{21-24}$ The intrachain 
part of the spectrum reproduces closely the spectrum of single Rouse chains and goes as $\tau^{-1 / 2}$. For longer times the relaxation spectrum obeys a power law, $\mathrm{H}(\tau) \sim$ $\tau^{-\alpha}$, related to an almost pure lattice relaxation. The exponent $\alpha$ depends on the lattice type considered, $\alpha=$ $\mathrm{d} / 2$, where $\mathrm{d}$ is the topological lattice dimension; $\alpha$ equals $3 / 2$ for $3 D$ cubic $c^{21,22}$ and 1 for $2 D$ square 23,24 lattices. Note that based on eq 6 , which relates $\mathrm{G}(\mathrm{t})$ to $\mathrm{H}(\tau)$, the form $\mathrm{H}(\tau) \sim \tau^{-\alpha}$ leads, by insertion, to $\mathrm{G}(\mathrm{t}) \sim$ $\mathrm{t}^{-\alpha}$, with the same value of $\alpha$. Furthermore, for these networks the maximal relaxation time of the Rouse chains between the vertexes and the minimal relaxation time of the lattice were found to be equal. ${ }^{21-24}$

We now return to our general case and focus on a (topologically) three-dimensional cubic lattice whose vertexes are connected by identical subunits of arbitrary topology. The elementary cell of the lattice is denoted by a three-component index $\boldsymbol{\Omega}=(\alpha, \beta, \gamma)$ where $\alpha, \beta$, and $\gamma$ range from 1 to $\mathrm{N}$. Such a cell contains a vertex and three subunits of $\mathrm{n}$ beads each; i.e., it consists of $\mathrm{s}$ $=3 n+1$ beads, which we number by the index $j \in$ $\{1 \ldots \mathrm{S}\}$. The whole network consists then of $\mathrm{SN}^{3}$ beads, numbered as $(\mathrm{j}, \boldsymbol{\Omega}) \equiv(\mathrm{j}, \alpha, \beta, \gamma)$. We can now simplify the determination of the eigenvalues of the connectivity matrix $\mathbf{A}$ of our system by making use of its topological symmetry. With hel p of the above notations, we rewrite the elastic term in the Langevin equations of motion (which is determined through the topology of the network) in the following way:

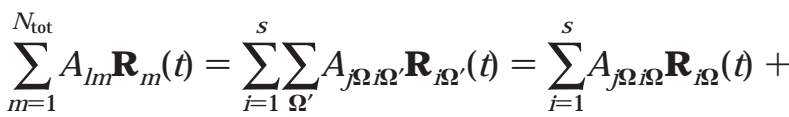

$$
\begin{aligned}
& \sum_{\mathrm{i}=1}^{\mathrm{s}} \sum_{\substack{\boldsymbol{\Omega}^{\prime} \\
\boldsymbol{\Omega}^{\prime} \neq \boldsymbol{\Omega}}} \mathrm{A}_{\mathrm{j} \mathbf{\Omega} \mathbf{i} \mathbf{\Omega}^{\prime}} \mathbf{R}_{\mathrm{i} \mathbf{\Omega}^{\prime}}(\mathrm{t})
\end{aligned}
$$

where we let $I \rightarrow(j, \boldsymbol{\Omega})$ and $m \rightarrow\left(i, \boldsymbol{\Omega}^{\prime}\right)$. Because of obvious symmetries, we may set in eq $18 \mathrm{~B}_{\mathrm{ji}}^{\text {(int) }} \equiv A_{j \boldsymbol{j} \mathbf{i} \Omega}$ and $\mathrm{B}_{\mathrm{ji}}^{(\text {ext) }}(\boldsymbol{\Delta}) \equiv \mathrm{B}_{\mathrm{ji}}^{\text {(ext) }}\left(\boldsymbol{\Omega}-\boldsymbol{\Omega}^{\prime}\right) \equiv A_{\mathrm{j} \boldsymbol{\Omega} i \boldsymbol{\Omega}^{\prime}}$, which leads to

$$
\sum_{m=1}^{\mathrm{N}_{\text {tot }}} \mathrm{A}_{\mathrm{Im}} \mathbf{R}_{\mathrm{m}}(\mathrm{t})=\sum_{\mathrm{i}=1}^{\mathrm{s}} \mathrm{B}_{\mathrm{ji}}^{(\mathrm{int})} \mathbf{R}_{\mathrm{i} \mathbf{\Omega}}(\mathrm{t})+\sum_{\mathrm{i}=1}^{\mathrm{s}} \sum_{\boldsymbol{\Delta}} \mathrm{B}_{\mathrm{ji}}^{(\mathrm{ext})}(\boldsymbol{\Delta}) \mathbf{R}_{\mathrm{i} \mathbf{\Omega}-\boldsymbol{\Delta}}(\mathrm{t})
$$

where $\boldsymbol{\Delta}=\boldsymbol{\Omega}-\boldsymbol{\Omega}^{\prime}$ is the relative distance between the lattice cells $\boldsymbol{\Omega}$ and $\boldsymbol{\Omega}^{\prime}$, measured in units of number of cells. In eq 19 the matrix $\mathbf{B}^{\text {(int) }}=\left\{B_{j i}^{(\text {int) }}\right\}$ is the connectivity matrix inside a given cell consisting of $s$ beads. In contrast, the matrix $B^{(\text {ext })}(\boldsymbol{\Delta})=\left\{B_{j i}^{(\text {ext })}(\boldsymbol{\Delta})\right\}$ provides the intercell connections: The nonzero elements of the matrices $B_{j i}^{(\text {ext) }}(\boldsymbol{\Delta})$ have as values negative integers; a value $(-1)$ for $B_{j i}^{\text {(ext) }}(\boldsymbol{\Delta})$ indicates that the total number of connections between bead j of cell $\boldsymbol{\Omega}$ and bead i of cell $\boldsymbol{\Omega}^{\prime}=\boldsymbol{\Omega}-\boldsymbol{\Delta}$ equals I. In the case of a regular lattice built from subunits in the manner of Figure 1, the sum over $\boldsymbol{\Delta}$ in eq 19 runs only over nearest-neighbor cells. Thus, in the 3D cubic network of Figure 1 the cell $\mathbf{\Omega}=$ $(\alpha, \beta, \gamma)$ is directly connected to its following six neighbors: $(\alpha-1, \beta, \gamma),(\alpha+1, \beta, \gamma),(\alpha, \beta-1, \gamma),(\alpha$, $\beta+1, \gamma),(\alpha, \beta, \gamma-1)$, and $(\alpha, \beta, \gamma+1)$; hence, $\Delta \in\{(1$, $0,0),(-1,0,0),(0,1,0),(0,-1,0),(0,0,1),(0,0,-1)\}$.

Because of the symmetry inherent in eq 19, the diagonalization of the connectivity matrix $\mathbf{A}$ of the network now simplifies. From a formal point of view the situation is very close to solid-state physics (Bravais lattice with a basis). However, one should note that in the arguments leading to eq 19 only the connectivity (a topological feature) entered. Hence, the networks considered here are not required to be translationally invariant. As an example, the positions of the beads $(\mathrm{j}, \boldsymbol{\Omega})$, $(\mathrm{j}, \boldsymbol{\Omega}+\boldsymbol{\Delta})$, and $(\mathrm{j}, \boldsymbol{\Omega}+\mathbf{2 \Delta})$ are not related by a single translation operator, as would be (in first-order) the case in a crystal. Here we have a GGS consisting of flexible subunits, whose conformations change widely under the underlying random Brownian forces and hence differ much from cell to cell. Nevertheless, using eq 19, we can proceed quite formally: We apply periodic boundary conditions to the whole GGS network and hence look for solutions to our Langevin equations of the form

$$
\begin{array}{r}
\mathbf{R}_{\mathbf{j} \boldsymbol{\Omega}}(\mathrm{t}) \equiv \mathbf{R}_{\mathrm{j} \alpha \beta \gamma}(\mathrm{t})=\sum_{\mathrm{k}_{1}, \mathrm{k}_{2}, \mathrm{k}_{3}} \mathbf{c}_{\mathbf{j k}} \exp \left(\mathrm { i } \left[\mathrm{k}_{1} \alpha+\mathrm{k}_{2} \beta+\right.\right. \\
\left.\left.\mathrm{k}_{3} \gamma\right]\right) \exp \left(-\lambda(\mathbf{k}) \mathrm{t} / \tau_{0}\right)
\end{array}
$$

In eq $20 \mathrm{i}$ is the imaginary unit, $\tau_{0}=\xi / \mathrm{K}$ is a characteristic (mi nimal) relaxation time of the network system, the $\mathbf{C}_{\mathbf{j k}}$ are constants, and the $\mathbf{k}=\left(\mathrm{k}_{1}, \mathrm{k}_{2}, \mathrm{k}_{3}\right)$ obey

$$
\mathrm{k}_{1}=\frac{2 \pi \mathrm{m}_{1}}{\mathrm{~N}}, \quad \mathrm{k}_{2}=\frac{2 \pi \mathrm{m}_{2}}{\mathrm{~N}}, \text { and } \mathrm{k}_{3}=\frac{2 \pi \mathrm{m}_{3}}{\mathrm{~N}}
$$

where the integers $m_{1}, m_{2}$, and $m_{3}$ range from 0 to ( $N$ - 1). Furthermore, the $\lambda(\mathbf{k})$ denote the different eigenvalues as functions of the parameter $\mathbf{k}$. Distinct from solid-state physics, however, here the three-component vector $\mathbf{k}$ simply counts the eigenvalues (modes) and is not related to a reciprocal wave vector.

Inserting eqs 20 and 19 into the Langevin equations of motion leads to

$$
\lambda(\mathbf{k}) \mathbf{C}_{\mathbf{j k}}=\sum_{\mathrm{i}=1}^{\mathrm{s}} \mathrm{B}_{\mathrm{ji}}(\mathbf{k}) \mathbf{C}_{\mathbf{i k}}
$$

where the elements of the matrix $\mathbf{B}(\mathbf{k})=\left\{\mathrm{B}_{\mathrm{ji}}(\mathbf{k})\right\}$ are given by

$$
\mathrm{B}_{\mathrm{ji}}(\mathbf{k})=\mathrm{B}_{\mathrm{ji}}^{(\mathrm{int})}+\sum_{\boldsymbol{\Delta}} \mathrm{B}_{\mathrm{ji}}^{(\mathrm{ext})}(\boldsymbol{\Delta}) \exp (-\mathrm{i} \mathbf{k} \cdot \boldsymbol{\Delta})
$$

We remark that the matrices $\mathbf{B}(\mathbf{k})$ include now all the information concerning thetopology (connectivity) inside each cell and also the way in which the cells are connected to each other. There are $\mathrm{N}^{3}$ different $\mathbf{B}(\mathbf{k})$ matrices, in which the exp $(-\mathbf{i k} \cdot \boldsymbol{\Delta})$ terms describe the phase shifts between the lattice cells. According to eq 22 , the problem of finding the eigenvalues of the connectivity matrix $\mathbf{A}$, which is a $\left(\mathrm{sN}^{3} \times \mathrm{sN}^{3}\right)$ matrix, is now reduced to the related problem of determining the eigenvalues of $\mathrm{N}^{3}$ different $(\mathrm{s} \times \mathrm{s})$ matrices.

We close this section by stressing that the derivation of eq 22 presented here is general and that it holds for an arbitrary topological structure of the subunits involved. Moreover, the 3D cubic lattice considered here is only a particular example of a regular lattice. Our approach can be easily extended to other lattice types. For instance, all the results of this section are valid also for a 2D square lattice built from complex subunits; to describe it one simply has to set $k_{3}=0$ in all the k-dependent equations established so far.

\section{Numerical Calculations: 2D Regular Lattice Built from SWR N Subunits}

Now we apply our general approach to one particular example of a network model, which allows us to combine 


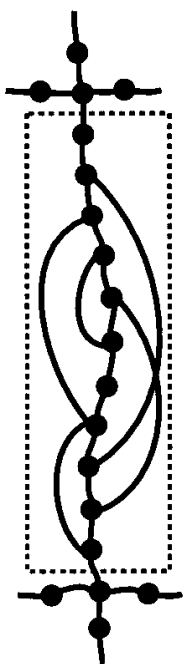

Figure 2. A subunit of particular interest: a small-world Rouse network (SWRN); see text for details.

high local disorder and mesoscopic order. In the case when cross-linking occurs in very dilute solutions of long flexible macromolecules, different polymer chains may be treated fairly independently of each other. Because of the stochastic features involved (random-walk-type configurations, Gaussian stochastic forces, etc.), monomers distant along the chain backbone may get cl ose in space; then, by means of irradiation such monomers can be chemically cross-linked. It follows that in very dilute solutions cross-link agglomerations are formed mostly from the individual chains, whose (originally distant) monomers get to be cross-linked. On the other hand, this type of cross-link is the hallmark of newly introduced connected structures, the so-called small-world Rouse networks (SWRN). ${ }^{25}$

In general, small-world networks (SWN) 26,27 are built from an underlying ordered lattice (say one-dimensional), to which one adds (with probability q) additional bonds between randomly chosen points. One intriguing property of 1D SWNs consists of the appearance of a so-called pseudogap 28 in the density of states, $\rho(\lambda)$, which goes as $\rho(\lambda) \sim \lambda^{-1 / 2} \exp \left[-\mathrm{C} / \lambda^{1 / 2}\right]$ for small $\lambda^{\prime}$ s. This means that the probability to find nonvanishing eigenvalues very close to 0 is extremely small. Small-world Rouse networks (SWRN) are now built from SWNs, by taking the sites to be Rouse beads and the bonds to be Rouse springs. From a theoretical point of view, we find the SWNs particularly useful, since each class of SWNs is determined by a single q parameter. In this work we will focus on such basic SWNs; whether adding bonds according to more complex distribution probabilities (i.e., by accounting for dependences on mutual distances, for correlations in adding bonds, etc.) leads to a more realistic pictures than the basic SWNs is a topic of recent debate. ${ }^{29,30}$

We take thus the lattice subunits to be 1D SWRNs (see Figure 2) and study the mechanical and dielectric response of a 2D square lattice composed of such SWRNs. In the SWRN construction we start from a linear Rouse chain consisting of $n$ beads connected by elastic springs. Then we connect (by means of additional springs) each bead of the chain in a random way (with probability $q / n$ ) to some other bead (cf. ref 25); see Figure 2. This leads to one specific, q-dependent SWRN realization. Finally, we use this SWRN realization as

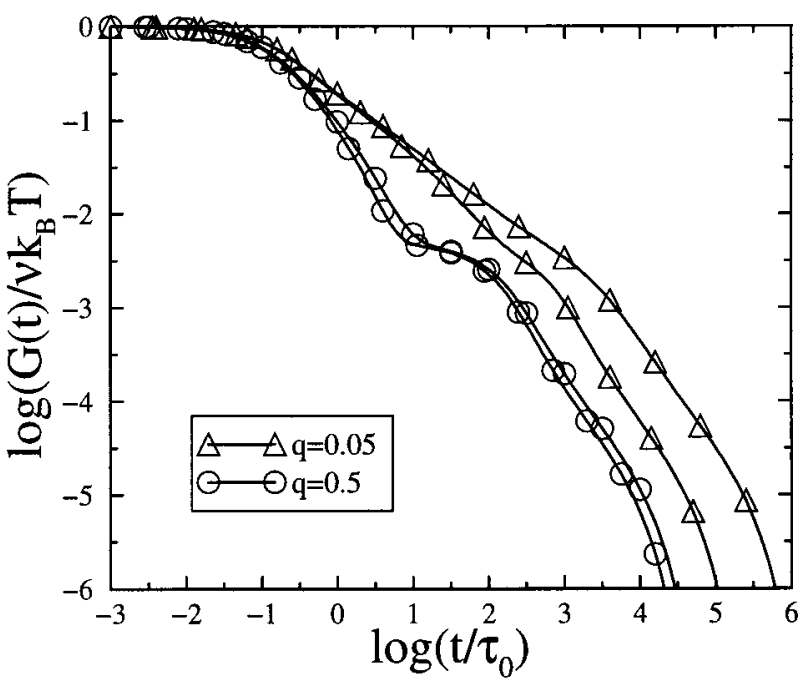

Figure 3. Reduced relaxation modulus $\mathrm{G}(\mathrm{t}) / \nu \mathrm{k}_{\mathrm{B}} \mathrm{T}$ plotted in double-logarithmic scales vs the reduced time $t / \tau_{0}$. Shown are results for two extreme realizations (out of ten) for SWRNbuilt networks with $q=0.05$ and $q=0.5$; see text for details.

subunits of a 2D $\mathrm{N} \times \mathrm{N}$ square lattice, obtaining our GGS.

The knowledge of the GGS leads directly to the matrix A, which has to be diagonalized; as noted in the previous section, however, we need only to diagonalize its corre sponding $\mathrm{N}^{2} \mathbf{B}(\mathbf{k})$ matrices and thus determine their eigenvalues. F or this we use standard routines ${ }^{31}$ for the diagonalization of symmetric matrices and determine for each $\mathbf{B}(\mathbf{k})$ its $\mathbf{s}=(2 n+1)$ eigenvalues. As parameters we take in the following calculations $n=200, N=20$ and vary $q$ and the number of SWRN realizations.

We begin with the calculation of the time-dependent relaxation modulus G(t) for our GGS. To get an idea of how sample to sample fluctuations influence the results, we start to calculate $\mathrm{G}(\mathrm{t})$ for isolated single SWRNs, which are not yet embedded in the lattice. We focus both on rather small $(q=0.05)$ and on sufficiently large ( $q$ $=0.5$ ) values for the probability $\mathrm{q}$ and consider 10 different realizations of the SWRN. Then we use the two extremal G(t) plots to determine the "worst" SWRN realizations for each $\mathrm{q}$. These are then used as subunits of the 2D lattice, for which we again evaluate $\mathrm{G}(\mathrm{t})$.

In Figure 3 we plot in double-logarithmic scales the corresponding curves of the reduced (dimensionless) relaxation modulus $\mathrm{G}(\mathrm{t}) / v \mathrm{k}_{\mathrm{B}} \mathrm{T}$ vs the reduced time $\mathrm{t} / \tau_{0}$. As is evident, the differences between the curves of the extremal cases are rather small, and the shapes of the curves are very similar for the two realizations. The curves clearly show the existence of several domains: these are related to the initial relaxation which involves only a few beads, to the relaxation of individual SWRN subunits, to an intermediate domain, and to the dynamics of the underlying square lattice. The spread in the $\mathrm{G}(\mathrm{t})$ curves can be directly attributed to the individual SWRN realizations underlying the disor dered network. Moreover, with decreasing q the curves become smoother, but the spread due to different SWRN realizations increases. This finding reflects the growing importance of fluctuations for small $q$.

In Figure 4 we plot the relaxation modulus $\mathrm{G}(\mathrm{t})$ of disordered networks, averaged over 100 realizations of SWRN subunits. To display the dependence of $\mathrm{G}(\mathrm{t})$ on disorder (i.e., on q), we let q take the values $0,0.05,0.1$, 0.3 , and 0.8 . The curve $q=0$ in Figure 4 corresponds to 


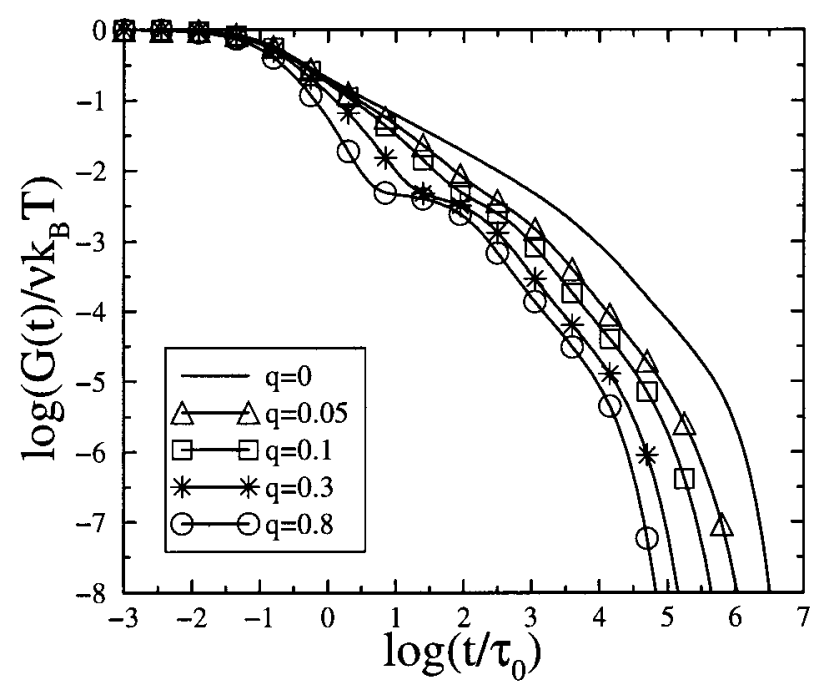

Figure 4. Dimensionless relaxation modulus $\mathrm{G}(\mathrm{t}) / v \mathrm{k}_{\mathrm{B}} \mathrm{T}$ vs the reduced time $t / \tau_{0}$ in double-logarithmic scales. Shown are results for SWRN-built networks with $q=0, q=0.05, q=$ $0.1, q=0.3$, and $q=0.8$. The averaging is performed over 100 distinct SWRN realizations.

the standard linear Rouse chain between lattice junctions. ${ }^{21-24}$ From Figure 4 one can distinguish very clearly, for $q=0$, several characteristic time ranges for $\mathrm{G}(\mathrm{t})$ : J ust after the initial domain, $\mathrm{t}<10^{-1} \tau_{0}$, one can see the region of internal relaxation of the Rouse chain between lattice vertexes, which extends up to $t<10^{3} \tau_{0}$; this domain is followed, up to times $\mathrm{t} \sim{ }^{1} 0^{6} \tau_{0}$, by the pure lattice relaxation. As remarked in the first paragraph of section 3 , for a linear Rouse chain $(d=1)$ and for a $2 \mathrm{D}$ regular lattice $(\mathrm{d}=2)$ the relaxation modulus scales, $G(t) \sim t^{-\alpha}$, where $\alpha=d / 2$. The crossover from the $t^{-1 / 2}$ to the $t^{-1}$ behavior can be clearly seen in Figure 4 for $\mathrm{q}=0$ at $\mathrm{t} \simeq 10^{3} \tau_{0}$. Finally, at even longer times one has a simple exponentional decay of $\mathrm{G}(\mathrm{t})$, a feature typical for GGSs of finite size.

We remark, based on Figure 4, that even a small amount of additional bonds introduced into the Rouse chain (i.e., $q \neq 0$ ) drastically changes the relaxation form from the simple chain $(q=0)$ behavior. With increasing $q$ the region of intra-subunit relaxation becomes smaller, because the rigidity of the subunits increases with $\mathrm{q}$. We note also that, in contrast to linear Rouse chains, small-world Rouse networks themselves represent an example of systems obeying a non-power-law behavior. ${ }^{25}$ Therefore, a simple picture involving several scaling regions (as found for $q=0$ ) is not observed for $q \neq 0$. M oreover, in the case of q large we observe in the region intermediate between intra-subunit and lattice relaxation a plateau-type behavior for $\mathrm{G}(\mathrm{t})$.

To gain a better understanding of the dynamic processes going on in the disordered network under study, we compare in Figure 5 for $q=0.8$ the relaxation modulus $\mathrm{G}(\mathrm{t})$ of a network built from SWRN subunits to the $\mathrm{G}(\mathrm{t})$ obtained for single SWRN subunits with free and fixed ends. From Figure 5 we infer that, after the internal relaxation of SWRN subunits comes to a close, the lattice relaxation barely starts, which means that at this point in time the lattice junctions are still immobile. We base this assertion on the appearance of a rather wide, plateau-type domain in the network's G(t) function. In this region (as is evident by inspection of Figure 5), the SWRN subunits of the network behave likeSWRNs with fixed end points; this means that there

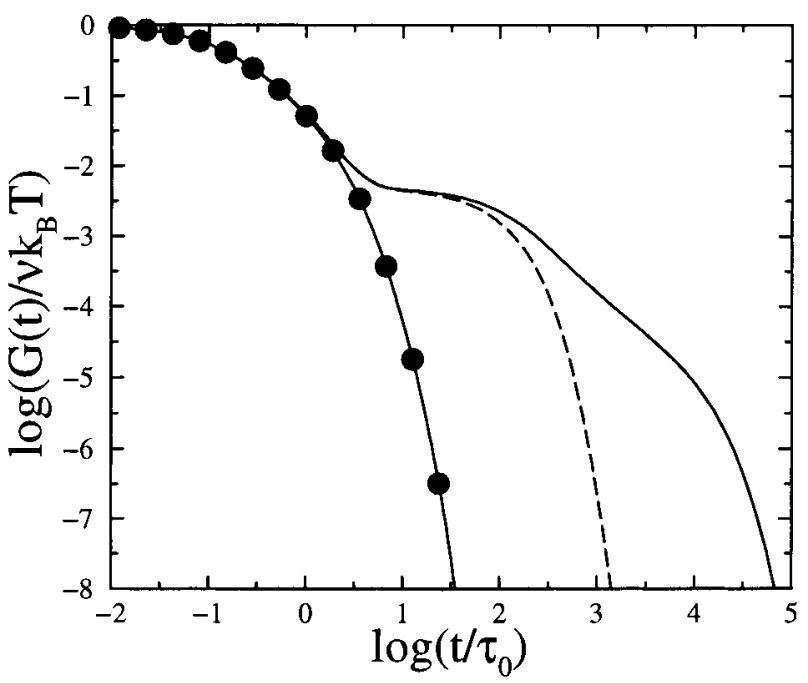

Figure 5. Dimensionless relaxation modulus $\mathrm{G}(\mathrm{t}) / v \mathrm{k}_{\mathrm{B}} \mathrm{T}$ vs the reduced time $t / \tau_{0}$ in double-logarithmic scales for $q=0.8$. Displayed are a single, free SWRN (circles), the same SWRN, but with fixed ends (dashed line), and the corresponding, 2D square lattice decorated with such SWRN subunits (solid line).

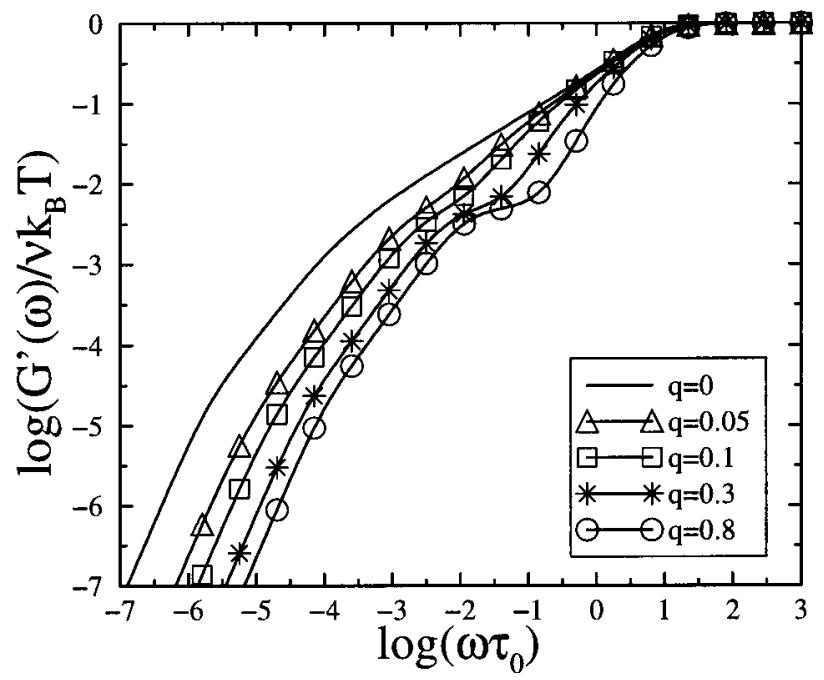

Figure 6. Reduced storage modulus $\mathrm{G}^{\prime}(\omega) / \nu \mathrm{k}_{\mathrm{B}} \mathrm{T}$ plotted in double-logarithmic scales vs the reduced frequency $\omega \tau_{0}$. Shown are results for networks with $q=0, q=0.05, q=0.1, q=0.3$, and $q=0.8$. The averaging is performed over 100 SWRN realizations.

is little lattice relaxation going on in this region. Such a $\mathrm{G}(\mathrm{t})$ behavior is characteristic for the existence of a gap in the relaxation spectrum of the network; the gap extends between the maximal relaxation time of a SWRN subunit and the minimal relaxation time of the lattice.

The same effects are also evident in the storage modulus $\mathrm{G}^{\prime}(\omega)$. We pl ot in Figure 6 for different q values $\mathrm{G}^{\prime}(\omega)$, obtained from averaging over 100 SWRN realizations. We again find that introducing even a small amount of additional springs into the linear Rousechain (i.e., going to SWRN ) changes the character of the $\mathrm{q}=$ 0 curve corresponding to the reqular situation. For large $q$ values $\mathrm{G}^{\prime}(\omega)$ displays again very clearly (now as a function of $\omega$ ) a plateau domain, a fact which discloses the existence of a gap in the density of states of the disordered network.

Similar conclusions can also be drawn from the loss modulus G" $(\omega)$, depicted in Figure 7. However, we find 


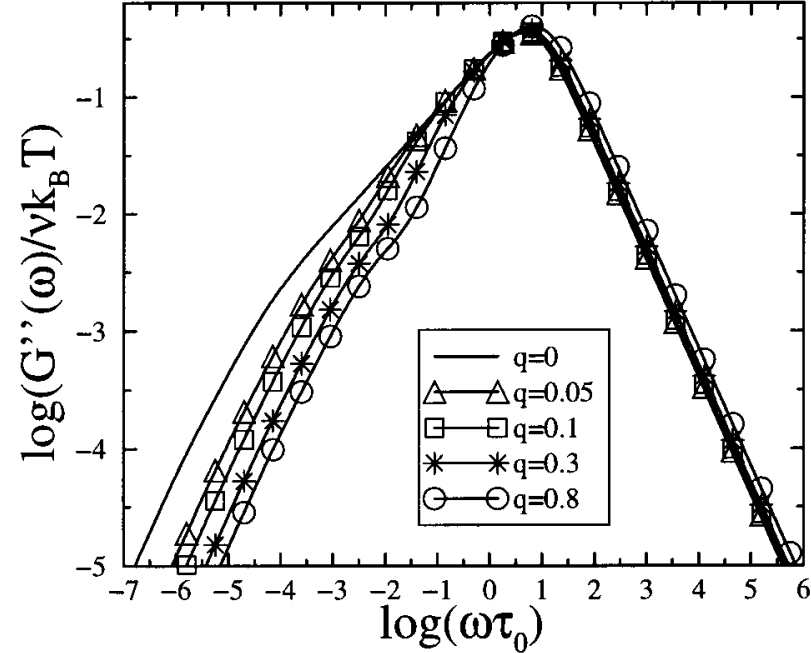

Figure 7. Reduced loss modulus $\mathrm{G}^{\prime \prime}(\omega) / v \mathrm{k}_{\mathrm{B}} \mathrm{T}$ plotted in doublelogarithmic scales vs the reduced frequency $\omega \tau_{0}$. Shown are results for SWRN-built networks with $q=0, q=0.05, q=$ $0.1, \mathrm{q}=0.3$, and $\mathrm{q}=0.8$. The averaging is performed over 100 SWRN realizations.

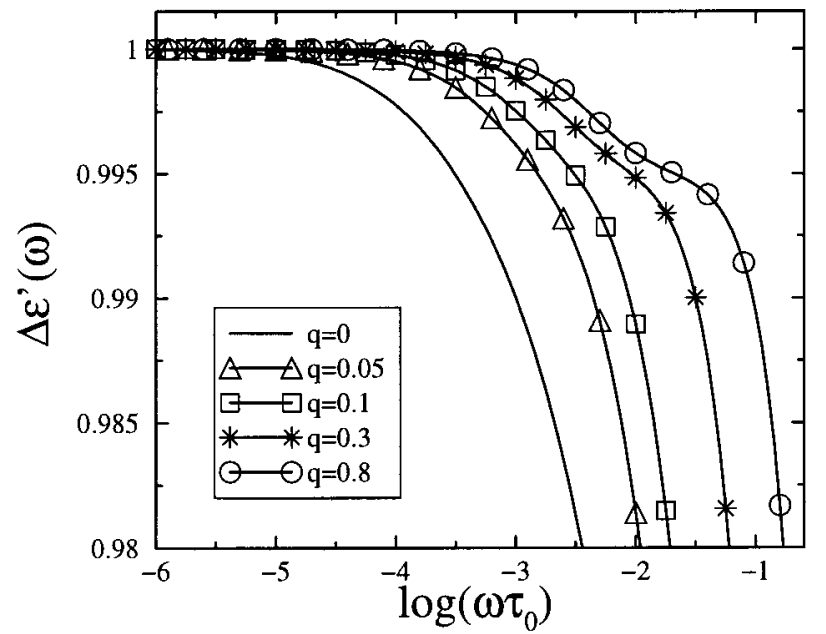

Figure 8. Real part of the dielectric susceptibility $\Delta \epsilon^{\prime}(\omega)$ plotted in semilogarithmic scales vs the reduced frequency $\omega \tau_{0}$. Shown are results for networks with $q=0, q=0.05, q=0.1$, $\mathrm{q}=0.3$, and $\mathrm{q}=0.8$. The averaging is performed over 100 SWRN realizations.

that the curves of $\mathrm{G}^{\prime \prime}(\omega)$ reflect less clearly than $\mathrm{G}^{\prime}(\omega)$ the aspects related to the network's disordered structure (cf. Figures 6 and 7). Mathematically, of course, $\mathrm{G}^{\prime}(\omega)$ and $\mathrm{G}^{\prime \prime}(\omega)$ carry the same information. Now, the quantity $\mathrm{G}^{\prime \prime}(\omega)$ corresponds to the loss of energy in the system and displays a maximum, whose position is mainly determined by rather high-frequency modes. Given that such modes involve just a few beads, their motion (and hence the maximum's position) depends only weakly on disorder (i.e., on q).

We end this exposition of numerical results with quantities related to dielectric relaxation. Given the connection between the mechanical and the dielectric relaxation forms, eqs 16 and 17, we refrain from displaying $\Delta \epsilon^{\prime \prime}(\omega)$ here, since the plots for the loss modulus $\mathrm{G}^{\prime \prime}(\omega)$ (Figure 7) and the dielectric loss factor $\Delta \epsilon^{\prime \prime}(\omega)$ differ only by a shift al ong the frequency axis. Turning to the real part of dielectric susceptibility $\Delta \epsilon^{\prime}(\omega)$, we display it in Figure 8 for different $q$ values. From Figure 8 one may deduce that with increasing $q$ the regions of intra-subunit relaxation become narrower and that they shift toward high frequencies; these facts are due to the increasing rigidity of the subunits with growing q. One may remark, for q large, the appearance in $\Delta \epsilon^{\prime}(\omega)$ of a steplike, plateau domain. A detailed analysis (not displayed here) shows unambiguously that this plateau is due to the above-mentioned gap in the relaxation spectrum. It follows that the gap can also be evidenced through dielectric relaxation measurements.

\section{Conclusions}

The main goal of this paper was to understand the role of strong structural inhomogeneities on the dynamic properties of cross-linked polymers. On the basis of recent experimental data, we confined our study to a special class of disorder, namely to the presence of crosslink agglomerations (clusters) in polymer networks and gels. To this purpose we put forward a model, which allows to combine local aspects of disorder with features of mesoscopic order: We focused on (topologically) regular lattices, whose vertexes are connected by complex subunits. Such models take into account the disorder inside the cross-link agglomerations through the subunits and also reflect, through the regular lattice, the mesoscopically rather homogeneous character of the samples. On the basis of the Rouse model and on its GGS extensions, we displayed the response functions of such systems under external fields, situations of much experimental relevance. Exemplarily, we applied the formalism to networks built from highly disodered subunits (small-world Rouse networks, SWRN). We found that for such networks the mechanical and dielectric responses are very sensitive to disorder: Networks built from SWRN subunits show interesting relaxation features and an unusual plateau-type behavior in the frequency (time) domain intermediate between the modes of the subunits and those of the regular lattice. As pointed out, this behavior is related to the existence of a gap in the relaxation spectrum of SWRN-built networks. To our knowledge, such features have not yet been highlighted in the analysis of experiments performed so far. The theoretical results derived in this paper may serve as an incentive to look car efully for such features in future experimental efforts on the dynamics of disordered polymer networks and gels. Our numerical evaluations show that several features of such (locally highly disordered and mesoscopically rather homogeneous) media are amenable to detection through mechanical and dielectric relaxation experiments.

Acknowledgment. The authors acknowledge the support of the Al exander von Humboldt Foundation, of the DFG, and of the Fonds der Chemischen Industrie.

\section{Appendix}

We have to calculate the normalized autocorrelation function of the total dipole moment $\mathrm{C}_{0}(\mathbf{M} ; \mathrm{t})$ of the GGS, eq 9. We start with the expression for $\langle\langle\mathbf{M}(0) \mathbf{M}(\mathrm{t})\rangle\rangle_{\text {or }}$, eq 11, which can be rewritten as

$$
\begin{aligned}
& \langle\langle\mathbf{M}(0) \mathbf{M}(\mathrm{t})\rangle\rangle_{\text {or }}= \\
& \mu^{2} \sum_{\mathrm{j}=1}^{N_{\mathrm{b}}}\left\langle\left(\mathbf{R}_{\uparrow}^{(\mathrm{j})}(0)-\mathbf{R}_{\mathrm{m}}^{(\mathrm{j})}(0)\right)\left(\mathbf{R}_{\uparrow}^{(\mathrm{j})}(\mathrm{t})-\mathbf{R}_{\mathrm{m}}^{(\mathrm{j})}(\mathrm{t})\right)\right\rangle
\end{aligned}
$$

since the vector of thejth bond between the Ith and the mth GGS beads is $\mathbf{P}_{\mathrm{j}}=\mathbf{R}_{\mathrm{l}}^{(\mathrm{j})}-\mathbf{R}_{\mathrm{m}}^{(\mathrm{j})}$. Here the subscript 
index (j) means that the Ith and the mth GGS beads belong to the jth bond. Let $\mathbf{S}=\left\{S_{\mathrm{I}}\right\}$ be the matrix that relates the normal modes $\mathbf{Q}_{k}(t)$ of the GGS to the corresponding coordinates $\mathbf{R}_{\mathbf{l}}(\mathrm{t})$ :

$$
\mathbf{R}_{\perp}^{(\mathrm{j})}(\mathrm{t})=\sum_{\mathrm{k}} \mathrm{S}_{I \mathrm{k}}^{(\mathrm{j})} \mathbf{Q}_{\mathrm{k}}(\mathrm{t})
$$

The matrix $\mathbf{S}$ also reduces the connectivity matrix $\mathbf{A}$ of the GGS to diagonal form. In other words, under this transformation the Langevin equations of motion, eq 1 , simplify to

$$
\xi \frac{\mathrm{d} \mathbf{Q}_{\mathrm{k}}(\mathrm{t})}{\mathrm{dt}}+\mathrm{K} \lambda_{\mathrm{k}} \mathbf{Q}_{\mathrm{k}}(\mathrm{t})=\xi \mathbf{W}_{\mathrm{k}}(\mathrm{t})
$$

where $\lambda_{k}$ is the kth eigenvalue of the connectivity matrix $\mathbf{A}$ and $\zeta \mathbf{W}_{\mathrm{k}}(\mathrm{t})$ is the normal mode transform of the thermal noise; we omit for simplicity the external force $\mathbf{F}(\mathrm{t})$.

Inserting the normal mode transformation, eq A2, into eq $A 1$ leads to

$$
\langle\langle\mathbf{M}(0) \mathbf{M}(\mathrm{t})\rangle\rangle_{\mathrm{or}}=\mu^{2} \sum_{\mathrm{k}=1}^{\mathrm{N}_{\text {tot }}}\left\langle\mathbf{Q}_{\mathrm{k}}{ }^{2}\right\rangle \exp \left(-\mathrm{t} / \tau_{\mathrm{k}}\right) \sum_{\mathrm{j}=1}^{\mathrm{N}_{\mathrm{b}}}\left(\mathrm{S}_{\mathrm{Ik}}^{(\mathrm{j})}-\mathrm{S}_{\mathrm{mk}}^{(\mathrm{j})}\right)^{2}
$$

where we use the orthogonality of the normal modes and also their simple time behavior (i.e., that each normal mode $\mathbf{Q}_{k}$ decays exponentially with the relaxation time $\left.\tau_{\mathrm{k}}=\tau_{0} / \lambda_{\mathrm{k}}\right)$ :

$$
\begin{aligned}
\left\langle\mathbf{Q}_{\mathrm{k}}(0) \mathbf{Q}_{\mathrm{k}^{\prime}}(\mathrm{t})\right\rangle=\delta_{\mathrm{kk}}\left\langle\mathbf{Q}_{\mathrm{k}}(0) \mathbf{Q}_{\mathrm{k}}(\mathrm{t})\right\rangle= \\
\delta_{\mathrm{kk}^{\prime}}\left\langle\mathbf{Q}_{\mathrm{k}}{ }^{2}\right\rangle \exp \left(-\mathrm{t} / \tau_{\mathrm{k}}\right)=\delta_{\mathrm{kk}^{\prime}}\left\langle\mathbf{Q}_{\mathrm{k}}{ }^{2}\right\rangle \exp \left(-\lambda_{\mathrm{k}} \mathrm{t} / \tau_{0}\right)
\end{aligned}
$$

With the use of eq $\mathrm{A} 3$ it is a straightforward matter to demonstrate that

$$
\left\langle\mathbf{Q}_{\mathrm{k}}{ }^{2}\right\rangle=\frac{\mathrm{k}_{\mathrm{B}} \mathrm{T}}{\mathrm{K} \lambda_{\mathrm{k}}}
$$

See, e.g. refs 21 and 22 for details. Hence, we need only to calculate the sum over $\mathrm{j}$ in eq A4. For this purpose we analyze in more detail the transformation of the potential energy $U(\{\mathbf{R}\})$ of the GGS, which enters in the Langevin equation, eq 12 . The normal mode transformation, eq A2, reduces the potential energy $U(\{\mathbf{R}\})$, eq 12, to diagonal form, i.e.

$$
\begin{array}{r}
\mathrm{U}(\{\mathbf{R}\})=\frac{\mathrm{K}}{2} \sum_{\mathrm{j}=1}^{\mathrm{N}_{\mathrm{b}}} \mathbf{P}_{\mathrm{j}}{ }^{2}=\frac{\mathrm{K}}{2} \sum_{\mathrm{k}=1}^{\mathrm{N}_{\text {tot }}}\left\langle\mathbf{Q}_{\mathrm{k}}{ }^{2}\right\rangle \sum_{\mathrm{j}=1}^{\mathrm{N}_{\mathrm{b}}}\left(\mathrm{S}_{\mathrm{lk}}^{(\mathrm{j})}-\mathrm{S}_{\mathrm{mk}}^{(\mathrm{j})}\right)^{2}= \\
\frac{\mathrm{K}^{\mathrm{N}_{\text {tot }}} \sum_{\mathrm{k}=1} \lambda_{\mathrm{k}}\left\langle\mathbf{Q}_{\mathrm{k}}{ }^{2}\right\rangle}{}
\end{array}
$$

Therefore, one has immediately

$$
\sum_{j=1}^{N_{b}}\left(S_{l k}^{(j)}-S_{m k}^{(j)}\right)^{2}=\lambda_{k}
$$

Inserting eqs A6 and A8 into eq A4 finally brings the quantity $\langle\langle\mathbf{M}(0) \mathbf{M}(\mathrm{t})\rangle\rangle_{\text {or }}$ into the following form:

$$
\langle\langle\mathbf{M}(0) \mathbf{M}(\mathrm{t})\rangle\rangle_{\text {or }}=\mu^{2} \frac{\mathrm{K}_{\mathrm{B}} \mathrm{T}_{\mathrm{K}}^{\mathrm{N}_{\text {tot }}}}{\mathrm{K}} \sum_{\mathrm{i}=1} \exp \left(-\lambda_{\mathrm{i}} \mathrm{t} / \tau_{0}\right)
$$

From this expression it follows that

$$
\mathrm{C}_{0}(\mathbf{M} ; \mathrm{t})=\frac{\langle\langle\mathbf{M}(0) \mathbf{M}(\mathrm{t})\rangle\rangle_{\text {or }}}{\left\langle\left\langle\mathbf{M}^{2}(0)\right\rangle\right\rangle_{\text {or }}}=\frac{1}{\mathrm{~N}_{\text {tot }}} \sum_{\text {tol }}^{\mathrm{N}_{\text {tot }}} \exp \left(-\lambda_{\mathrm{i}} \mathrm{t} / \tau_{0}\right)
$$

i.e. eq 13.

\section{References and Notes}

(1) Bastide, J .; Leibler, L.; Prost, J . Macromolecules 1990, 23, 1821.

(2) Mendes, E.; Lindner, P.; Buzier, M.; Boué, F.; Bastide, J. Phys. Rev. Lett. 1991, 66, 1595.

(3) Wun, K. L.; Prins, W. J . Polym. Sci., Polym. Phys. Ed. 1974, $12,533$.

(4) Hecht, A. M.; Duplessix, R.; Geissler, E. Macromolecules 1985, 18, 2167.

(5) Lindemann, B.; Schröder, U. P.; Oppermann, W. Macromolecules 1997, 30, 4073.

(6) Ferry, J. D. Viscoelastic Properties of Polymers, 3rd ed.; Wiley: New York, 1980.

(7) Sommer, J.-U.; Blumen, A. J . Phys. A 1995, 28, 6669.

(8) Schiessel, H. Phys. Rev. E 1998, 57, 5775.

(9) Rouse, P. E. J . Chem. Phys. 1953, 21, 1272.

(10) Doi, M.; Edwards, S. F. The Theory of Polymer Dynamics; Clarendon: Oxford, 1986.

(11) Kittel, C. Introduction to Solid StatePhysics, 6th ed.; Wiley: New York, 1986.

(12) Ashcroft, N. W.; Mermin, N. D. Solid State Physics; Sanders College: Philadel phia, 1981.

(13) Ziman, J. M. Principles of the Theory of Solids, 2nd ed.; Cambridge University Press: Cambridge, 1972.

(14) Biswas, P.; Kant, R.; Blumen, A. Macromol. Theory Simul. 2000, 9, 56.

(15) Williams, G. Chem. Rev. 1972, 72, 55.

(16) Stockmayer, W. H.; Baur, M. E. J . Am. Chem. Soc. 1964, 86, 3485.

(17) Stockmayer, W. H. Pure Appl. Chem. 1967, 15, 539

(18) Yamakawa, H. Modern Theory of Polymer Solutions; Harper and Row: New York, 1971.

(19) Gotlib, Yu. Ya.; Gurtovenko, A. A. Macromol. Theory Simul. 1996, 5, 969.

(20) Blumen, A.; Gurtovenko, A. A.; J espersen, S. J . Non-Cryst. Solids, in press.

(21) Gurtovenko, A. A.; Gotlib, Yu. Ya. Macromolecules 1998, 31, 5756.

(22) Gurtovenko, A. A.; Gotlib, Yu. Ya. Macromol ecules 2000, 33, 6578.

(23) Gotlib, Yu. Ya.; Gurtovenko, A. A. Macromol. Theory Simul. 2000, 9, 407.

(24) Gurtovenko, A. A.; Gotlib, Yu. Ya. Macromol. Theory Simul. 2000, 9, 416.

(25) J espersen, S.; Sokolov, I. M.; Blumen, A. J . Chem. Phys. 2000, $113,7652$.

(26) Watts, D. J .; Strogatz, S. H. Nature(London) 1998, 393, 440.

(27) Watts, D. J . Small Worlds: The Dynamics of Networks between Order and Randomness; Princeton University Press: Princeton, 1999.

(28) Monasson, R. Eur. Phys. J . B 1999, 12, 555.

(29) J espersen, S.; Blumen, A. Phys. Rev. E 2000, 62, 6270.

(30) Sen, P.; Chakrabarti, B. K. J . Phys. A 2001, 34, 7749.

(31) Press, W. H.; Flannery, B. P.; Teukolsky, S. A.; Vetterling, W. T. Numerical Recipes in C. The Art of Scientific Computing; Cambridge University Press: New York, 1988.

MA011851+ 